\title{
DYNAMIC FACTORS IN THE FORMATION AND RE-ABSORPTION OF AQUEOUS HUMOUR \\ BY
}

\author{
JONAS S. FRIEDENWALD*
}

THE recent article by Duke-Elder and Davson, ${ }^{1}$, discussitig the work on aqueous humour dynamics by Kinsey, Grant and Cogan, ${ }^{2}$ provides the opportunity for an exchange of views on this subject; which, I believe, will show that the area of agreement among workers in this field is now very much greater than the area still in dispute. There is apparently general agreement that some exchange of constituents occurs between blood and aqueous by a group of processes which may be summed together as diffusion, and that superimposed upon this equilibrating trend there are in addition dynamic factors which prevent the development of complete equilibrium between blood and aqueous. Duke-Elder and Davson sum these dynamic factors together as secretion. Kinsey and Grant attempt to separate the dynamic factors into secretion and leak. The problem of determining the factors responsible for these deviations from equilibrium can also be approached from a detailed analysis of the secreting and re-absorbing mechanism, and it is this approach that I have followed.

So far as concerns the diffusional exchange between blood and aqueous, the determining factors must be, on the one hand the size, mobility, ionic charge, etc., of each component that is exchanged, and on the other hand, the membrane characteristics or permeabilities of the several barriers across which exchange takes place. It seems obvious that these several barriers do not have identical permeabilities. The ciliary barrier is very different from that in the iris, as has been shown by many investigators. Kinsey and Grant's hypothesis of a leakage of aqueous into Schlemm's canal is one particularised assumption that the permeability of this portion of the blood-aqueous barrier differs from that in the iris and ciliary body. There is, in fact, no reason for assuming that the permeability for each individual component of the aqueous varies in the same fixed proportion in all the different parts of the blood-aqueous barrier.

Kinsey and Grant have. measured the rate of transfer from blood to aqueous of the various components of the intraocular fluid, and from these measurements have computed co-efficients of transfer for each of the substances studied. Such computed co-efficients, however, must represent only averages for

* From the Wilmer Ophthalmological Institute of the Johns Hopkins University School of Medicine. Received for publication. May 27; 1944. 
the whole complex system, averages in which the proportion contributed by each part of the blood-aqueous barrier presumably varies for each component of the aqueous. 'Kinsey and Grant have indeed 'recognised this, and have concluded that a secretory mechanism is required to account for the observed transfer rates of electrolytes, while diffusion alone may account for the transfer rates of water and of non-electrolytes. Their studies were not primarily concerned with what tissues contributed to these several mechanisms nor how these mechanisms operate.

Duke-Elder and his co-workers, on the other hand, have directed their energies not toward the rate of transfer of individual components from blood to aqueous, but to the measurement of the concentration ratios of different components in blood and aqueous. They have shown that the aqueous is hypertonic to blood plasma, that it contains a considerable excess of electrolytes when compared with plasma dialysate. They conclude, in agreement with Kinsey and Grant, that secretory energy is required to maintain this discrepancy. They have not been primarily concerned with how secretory and diffusional forces may co-operate in the total result.

What, then, do we actually know about the secretory and reabsorptive mechanisms, and to what extent does our knowledge of these mechanisms now suffice to explain the observed rates of transfer and concentration ratios of various substances between the blood and aqueous?

The secretory mechanism in the ciliary processes has been found to be connected with the oxidative interchange between the epithelium and the stroma of this tissue. ${ }^{3}$ Such an oxidative interchange must lead to the production of anions in the epithelium, primarily hydroxyl ions formed from the reduction of oxygen, and the production of cations in the stroma, primarily hydrogen ions formed from the oxidation of hydrogen. Concentrating our attention for the moment on the epithelium, it is to be presumed that the newly-formed anionic charge would be transferred to other ionic species by buffer action. Thus, for instance, the reaction

$$
\overline{\mathrm{O}} \mathrm{H}+\mathrm{CO}_{2} \rightarrow \overline{\mathrm{H}} \mathrm{CO}_{3}
$$

may be supposed to describe the chief overall mechanism by which the disappearance of the hydroxyl ions is effected. The aqueous, however, does not contain an excess of bicarbonate. In order to account for this fact we must, therefore, assume an exchange of bicarbonate for other anions, chiefly chloride, an exchange such as has been shown to take place with great ease between red blood cells and plasma. There is no evidence of an excess of bicarbonate even in the posterior chamber. Consequently the exchange of bicarbonate for chloride must take place 


\section{FORMATION AND RE-ABSORPTION OF AQUEOUS HUMOUR 505}

chiefly in the ciliary body itself, and the stroma-epithelium barrier must permit such an exchange.

The possibility, of such an anionic exchange across the secretory barrier in the ciliary processes is a characteristic of the permeability of this barrier which had not previously been postulated; and for which no direct experimental support has as yet been obtained. Such an exchange is not necessarily a feature of every secretory mechanism. For instance, the saliva of cows is approximately isotonic with plasma in its electrolyte content and resembles the aqueous in many aspects of its composition, but its chief anion is bicarbonate, not chloride.

In the ciliary stroma, the excess hydrogen ions formed as a result of the oxidative interchange is likewise presumably disposed of by local buffering action of the tissue and by exchange of cations with the blood. In any case, a net excess of anions in the epithelium and of cations in the stroma still remains at this stage of the argument, this net excess to be balanced by an overall transfer from one tissue to the other. In the ciliary body, the barrier between epithelium and stroma is negatively charged. ${ }^{3}$ It is well established that the layer of water in contact with such a negatively charged membrane must contain an excess of cations held there by the electrical forces of the charge in the membrane. This water layer adsorbed on the surface of the membrane is, then, positively charged because of the excess of cations which it contains, and it will be repelled by the excess cations in the stroma, attracted by the excess anions in the epithelium. Bethe ${ }^{4}$ has, in fact, shown by direct experiment that the net ionic movement caused by the imposition of an electrical force across a charged membrane in a watery environment is furnished by the adsorbed layer, and that the fluid moved under such circumstances is hypertonic in comparison with the main body of fluid from which it is derived. Thus the portion of the aqueous which is " secreted " is the adsorbed layer on the secretory membrane.

As noted above, Duke-Elder and his co-workers ${ }^{5}$ have shown that the aqueous is hypertonic in respect to electrolytes as compared with the blood, and conclude that secretory forces are involved. From measurements of the rate of transfer from blood to aqueous, Kinsey and Grant also conclude that electrolytes are secreted. This may, then, be taken as further evidence that it is the hypertonic adsorbed layer on the ciliary membrane which is secreted. The fluid which is transported into the epithelium may, in fact, be much more hypertonic than the aqueous, for osmotic exchange may begin to reduce its hypertonicity as soon as it reaches the epithelium. This is, again, an aspect of the secretory mechanism for which we have as yet no direct experimental data. 'It is within the framework of such suppositions, 
however, that Kinsey and Grant's conclusion that electrolytes are secreted while water is moved by osmosis (in addition to exchange) may be understood.

Various workers (Walker, ${ }^{6}$ Adler, ${ }^{7}$ Benham $^{8}$ and others) have shown that the aqueous is hypotonic in respect to urea and some other non-electrolytes as compared with the blood. Kinsey and Grant confirmed this and attribute this to "ultra-filtration," or in more general terms to diffusion, assuming a differential permeability in favour of water and against non-electrolytes at the blood-aqueous barrier. Duke-Elder in his criticism of this work objects to the ultra-filtration in the absence of secretory activity. In order to filter fluid, as dilute in urea as is the aqueous, unphysiological levels of capillary pressure would be required in the absence of secretion. However, Kinsey and Grant have concluded that secretion of electrolytes takes place. (A minimum capillary pressure of $75 \mathrm{~m} . \mathrm{m}$. Hg. would be required to filter an intra-ocular fluid containing $10 \mathrm{~m} . \mathrm{gm}$. per cent. less urea than in the blood. This is approximately the normal pressure in the central retinal artery, and is $25-35 \mathrm{~m}: \mathrm{m}$. higher than estimates of intra-ocular capillary pressure based on other methods of study.) I believe that it is compatible with the arguments of both these investigators to point out that there are two ways in which aqueous hypotonic in non-electrolytes can be formed by the ciliary processes. First, it is quite possible that the adsorbed layer on the secretory membrane is itself relatively deficient in non-electrolytes. The more hydrophilic and less surface acting such substances are, the lower, proportionately, will be their concentration in the adsorbed layer. Evidence on this possibility might be obtained through a study of such substances as alcohol which have a higher tendency to be adsorbed.* At any rate, a deficiency of non-electrolytes on the secretory membrane would be a special kind of permeability factor such as Kinsey and Grant envisage. The energy required for the movement of the adsorbed layer would, in this case, be supplied by the secretory mechanism, not by ultra-filtration.

A second way in which the secretory apparatus could yield a fluid hypotonic in non-electrolytes is the following: As indicated above, the adsorbed layer on the secretory membrane is probably

* A number of investigators have shown that the concentration of alcohol in the spinal fluid tends to be higher than that in the blood. Mehrtens and Newman ${ }^{9}$, for instance, maintained the blood alcohol level stationary for 4-5 hours by continuous intravenous infusion. At the end of this period, the spinal and cysternal fluid levels were on the average 10 per cent. higher than in the blood. Part of this excess is to be accounted for by the fact that the conoentration of alcohol in plasma is higher than in whole blood, part by the fact that the water content of plasma is lower than that of spinal fuid. When allowances are made for these two corrections most investigators ${ }^{10}$ agree that the spinal fluid alcohol concentration is in equilibrium with that in plasma, - but there is in any case no deficit of alcohol in the spinal fluid as is the case in respect of urea. 
quite hypertonic in cations. When this fluid is transferred to the epithelium it is met by an equal excess of anions yielding a fluid presumably more hypertonic than the final aqueous. This fluid may now be diluted by osmotic forces. It has been shown that the secretory membrane is relatively impermeable to the passage of cations except under the influence of electrical, i.e., secretory, forces. ${ }^{3}$ It would follow that the dilution of this fluid could not occur by the diffusion of salt back into the stroma, but only by the diffusion of water from stroma to epithelium. Thermodynamic equilibrium would not be attained under these conditions, only a steady state, but the thermodynamic principle of " least work" may still be applicable, and the least work toward establishing that steady state would occur if fluid relatively hypotonic in non-electrolytes were transported. It is this concept which Kinsey and Grant favour. There are, therefore, sound thermodynamic grounds for believing that the fluid transported by this mechanism would be hypotonic in non-electrolytes. This corresponds also, I believe, to Duke-Elder's argument that energy must be supplied in order to produce such hypotonicity.

It would appear, then, that the components of the secretory apparatus so far identified, with a few additional postulated specifications, suffice to account for the formation of aqueous hypertonic in electrolytes and hypotonic in non-electrolytes. The energy both for the actual transport of the fluid and for its deviations from a dialysate is provided by the metabolic interaction between epithelium and stroma. In this sense the fluid transported may properly be called a secretion, though electrical and osmotic forces and membrane characteristics all enter into determining its final composition.

In the iris the blood-aqueous barrier consists only of capillary endothelium. There appears to be no evidence either in respect to the iris capillaries, or in respect to capillaries in ather organs that secretion across the capillary wall takes place. Hence we have no grounds for supposing that anything except diffusional exchange can take place in the iris portion of the blood-aqueous barrier. Consequently, the contribution which the iris makes to the composition of the aqueous must be to bring it nearer to equilibrium with the blood plasma; that is, to reduce its hypertonicity in electrolytes and its hypotonicity in non-electrolytes. No experimental data are available by which to determine whether the equilibrating contribution of the iris is large or small, but it is presumably not negligible.

Duke-Elder and his co-workers have shown that the total osmotic pressure of the aqueous is considerably higher than the total osmotic pressure, including, proteins, of the plasma. The osmotic forces acting across the blood-aqueous barrier in the iris 
would therefore move water from blood to aqueous. The hydrostatic pressure in the iris capillaries is certainly not less than the intra-ocular pressure. Thus the net water transfer across the iris barrier must be from blood to aqueous. This is in accord with Kinsey and Grant's conclusion that part of the water' transferred into the eye is moved by osmotic forces.

We have arrived, then, at a curious paradox. Duke-Elder's studies on the osmotic pressure of the aqueous, Kinsey and Grant's studies on the rates of transfer of the individual components of the aqueous, and my own investigations on the secretory mechanism all serve to explain in mutually compatible ways how extra water over and above the diffusional exchange enters the eye. How, then, is the volume of the eye maintained constant? Kinsey and Grant were the first to point out clearly that simple diffusion into the blood does not suffice to explain the exit of water from the eye, and have suggested leakage out of the eye as a possible way out of the dilemma.

The objections of Duke-Elder and Davson to the leak hypothesis do not seem to me to be valid. They argue that the composition of the aqueous deviates from that of the plasma dialysate by such a large factor that the hydrostatic energies of filtration plus leak do not suffice for the work to be performed, and hence that secretion is necessary. This is undoubtedly true, but the dilemma which the leak hypothesis was designed to resolve was reached only after the secretion of electrolytes into the eye had already been accepted as proven. It may be suggested that this dilemma could be equally resolved by assuming a secretion of water out of the eye. However, no organ for such secretion is apparent.

While the leak hypothesis is, then, compatible with the experimental data of Kinsey and Grant and also those of Duke-Elder, it is not the only hypothesis for the exit of intra-ocular fluid which is compatible, with these data, and there are some other data which give weight to some of the alternatives.

It may be pointed out, for instance, that Leber's conception that fluid filters out of the eye into Schlemm's canal does not involve any equilibrating osmotic factors between blood and aqueous at the aqueous-Schlemm boundary, and hence would be a satisfactory alternative to a leak. There is, in fact, no way to distinguish between filtration and leak through chemical analysis of the aqueous. Duke-Elder, ${ }^{11}$ however, has found that the pressure in the episcleral veins, with which Schlemm's canal is connected, is equal to or slightly higher than the intra-ocular pressure. If this is true, neither leak or filtration into Schlemm's canal is possible. How, then, can we escape the dilemma which Kinsey and Grant have formulated?

An alternative which is compatible with the latter's analysis is 
the slow trickle of plasma into Schlemm's canal, for the existence of which I have previously presented some evidence. ${ }^{12}$ In order to show that this is compatible with the requirements of Kinsey and Grant let me present the following illustration. If a limited volume of plasma is dialized against hypertonic salț solution through a collodion membrane, there is first a movement of water from the plasma and a simultaneous movement of salt into the plasma. After a period the salt concentration in the plasma compartment approaches that in the salt solution, and thereafter water and salt both move into the plasma compartment diluting the plasma proteins. When this stage has been established some of the diluted plasma can be removed and replaced by a small amount of fresh plasma. If the newly added plasma is given in sufficiently small amount, the osmotic transfer of fluid into the plasma compartment will not be interrupted. In fact, a continuous slow trickle of plasma and corresponding removal of diluted plasma can keep the absorption of fluid going as a steady process-all the while the absorbed fluid having a higher osmotic pressure than that of the plasma which is being added. Within limits, the rate of fluid absorption will vary with the rate of plasma supplied.

This is precisely the arrangement which histological studies have indicated is the case for Schlemm's canal, with its narrow and scanty afferent arterioles, its wide and numerous efferent venules, its content of few blood cells and diluted plasma. So far as concerns the absorption of non-isotonic aqueous, this mechanism can function as if 1 t were a leak, but the rate of absorption can be subject to vasomotor control.

Kinsey and Grant estimate that the rate of "leak " in rabbits is 4 cu. m.m. per minute, i.e., $1 \frac{1}{2}$ to 2 per cent. of the aqueous. This should be less than the rate of disappearance of crystalloids injected into the anterior chamber, since some of such injected crystalloids may diffuse out of the anterior chamber by other routes. The results obtained from such injection experiments are, however, somewhat lower-for instance, 1 per cent. per minute for glucose, 0.8 per cent. per minute for ascorbic acid. Whether this discrepancy indicates an error in the theory or is to be accounted for within the limits. of experimental error remains to be seen.

In summary, the picture of the secretory and reabsorptive mechanism which has grown out of the histological and histochemical studies which my colleagues and I have reported, is compatible with the findings of Duke-Elder and his co-workers in regard to the constitution of the aqueous, and with the findings of Kinsey and his co-workers on the velocity of exchange of components between blood and aqueous. Similarly, the theoretical 
conclusions which Duke-Elder and Kinsey draw from their studies are compatible with the details of structure and organisation which we have found in the ocular tissues.

\section{REFERENCES}

1. Duke-Elder and Davson (1943).-Brit. Jl. Ophthal., Vol. XXVII, p. 431.

2. KINŚey, Grant and Cogan (1942).-Arch. Ophthal., Vol. XXVII, p. 242. KINSEY, GRANT and COGAN, Livingood and CURTIS (1942).-Arch. Ophthal., Vol XXVII, p. 1126.

KINSEY and GRANT (1942).- $J l$. Gen. Physiol., Vol. XXVI, pp. 119, 131.

3. FriedenWALd and Stiehler (1938). -Arch. Ophthal., Vol. XX, p. 761

FRIEDENWALD, BUSCHKE and MICHEL (1943). -Arch. Ophthal., Vol. XXIX, p. 535.

4. Bethe and Toropoff (1915).-Zeitschr. f. Phys. Chem., Vol. XXXIX, p. 597.

5. DuKE-Elder, Quilílan and DavSON (1940):-Brit.Ji. Ophthal., Vol, XXIV,

p. 421 .
DAvSon and WeLd (1941)'-Amer. Jl. Physiol., Vol. CXXXIV, p. 1.

BenhaM, G. H., DUKE-Elder, W. S. and Hodgson, T. H. (1938),- $J l$. Physiol.,IVol. XCII, p. 355.

6. WAlker, A. M. (1933).-Jl. Biol. Chem:, Vol. CI, p. 269.

7. AdLER, F. H. (1933). -Arch. Ophthal., Vol. X, p. 11.

8. BeNHAM, G. H. (1937).-Biochem: Jl., Vol. XXXI, p. 1157.

9. Mehrtens and NeWman (1933).-Arch. Neurol. and Psych., Vol. XXX, p. 1092.

10. Goldberg (1937).-Skand. Arch. Physiol., Vol. LXXVII, p. 179.

Fleming and Stotz (1935).-Arch. Neurol. and Pysch., Vol. XXXIII p. 492. - (1936).-Arch. Neurol. and Psych., Vol. XXXV, p. 117.

11. DUKe-ElDER; W. S. (1926).-Brit. Jl. Ophthal., Vol. X, p. 513.

12. FriedenWaLd, J. S. (1936).-Arch. Ophthal., Vol. XVI, p. 65.

\section{MUCOCELE OF MAXILLARY ANTRUM}

BY

\section{G. E. DoDDS}

WEST AFRICAN MEDTCAL SERVICE

THE patient in this case presented himself at a native dispensary in the "bush" in Southern Nigeria.

All that could be done was to examine, photograph and endeavour to persuade him to come to hospital. Nothing further was attempted-as it was hoped that at some future date transillumination, nasal examination, X-ray and operation could be carried out.

At the time the case was seen it would have been quite easy to insert an exploratory needle through the skin of the lower eyelid and doubtless a thick gelatinous flutid could have been aspirated.

Such a procedure would have undoubtedly encouraged the patient to think that a cure had been effected or would eventuate in time, and so the possibility of further enquiries would have been indefinitely postponed. A knowledge of local psychology is essential for the benefit of all concerned, and curiosity must be tempered with discretion if medical secrets are to be exposed. 